Revista Internacional em Língua Portuguesa ISSN: 2182-4452 (impresso) / ISSN: 2184-2043 (eletrónico)

\title{
LABSIM - Experiência em Simulação como Metodologia de Ensino
}

\author{
Maria do Carmo Barros de Melo ${ }^{1}$ \\ Monalisa Maria Gresta ${ }^{1}$ \\ Nara Lucia Carvalho e Silva ${ }^{1}$ \\ Gimar Fidelis ${ }^{1}$ \\ Maria Angélica Tibaes ${ }^{1}$ \\ Josemar de Almeida Moura ${ }^{1}$ \\ Claudio de Souza ${ }^{1}$ \\ ${ }^{1}$ Universidade Federal de Minas Gerais, Brasil; E-Mail: mcbmelo@gmail.com; monalisagresta@yahoo.com.br; \\ carvalho.nara@gmail.com; fidelis@medicina.ufmg.br; mariaangelicaservadedeus@hotmail.com; \\ josemar.a.moura@gmail.com; csouza@medicina.ufmg.br.
}

\section{Resumo}

O ensino mediado por simulação é cada vez mais utilizado por instituições educacionais e serviços de saúde. É uma metodologia de ensino seguro e ético que complementa a prática adquirida com o paciente. Vários são os formatos e as técnicas de simulação, como, por exemplo, a utilização de manequins de baixa ou alta fidelidade, atores, simulação híbrida e háptica. Este artigo relata a experiência de criação e expansão do uso de simulação na Faculdade de Medicina da Universidade Federal de Minas Gerais, Brasil. As perspectivas futuras das tecnologias de informação e comunicação na área da saúde acopladas ao uso de simulação avançada e computacional são promissoras, especialmente, para treinamento de profissionais de saúde, estudantes de graduação e pós-graduação.

Palavras-chave: educação médica; educação em saúde; simulação; simulação de paciente.

\begin{abstract}
Simulation-mediated teaching is increasingly used by educational institutions and health services. It is a safe and ethical teaching methodology that complements the practice acquired with the patient. Several are the formats and the simulation techniques, for example with the use of mannequins of low or high fidelity, actors, hybrid simulation and haptics. This article reports the experience of creating and expanding the use of simulation in the Faculty of Medicine of the Federal University of Minas Gerais, Brazil. The future perspectives of information and communication technologies in the field of health coupled with the use of advanced and computational simulation are promising, especially for training health professionals, undergraduate and graduate students.
\end{abstract}

Keywords: education; health education; medical; patient simulation; simulation.

\section{Introdução}

O ensino mediado por simulação vem sendo aprimorado nos últimos anos, em especial no ensino médico. No Brasil, o Ministério da Educação tem como diretriz curricular do ensino médico a necessidade de utilização de laboratórios de simulação. A portaria faz referência ao aprendizado em situações e ambientes protegidos e controlados, ou em simulações da realidade, identificando e avaliando o erro, como insumo da aprendizagem profissional e organizacional e como suporte pedagógico, enfatizando a necessidade de treinamento em atendimentos utilizando equipes multidisciplinares (Brasil, 2014).

A prática simulada previamente ao atendimento do paciente é recomendado por diversas sociedades científicas e eticamente é mais aceitável, podendo ser utilizada para treinamento de habilidades em procedimentos 
constrangedores (como, por exemplo, toque retal ou vaginal), em trabalho em equipe ou atendimento de urgências, entre outros.

No ensino do adulto, a andragogia se fundamenta na necessidade de motivação para que o processo ensino/aprendizagem ocorra, na contextualização, prática clínica, no compartilhamento de experiências. A missão do professor é auxiliar o aluno a atingir o seu melhor potencial (Dent, Harden, \& Hunt, 2017). É importante buscar a possibilidade de tornar o ensino mais efetivo e prazeroso (Martins et al., 2012).

A Faculdade de Medicina (FM) da Universidade Federal de Minas Gerais (UFMG) vem utilizando práticas simuladas para o ensino médico desde 1975, com investimento financeiro e logístico crescente no decorrer dos anos. É a que tem mais alunos matriculados por ano no país (320 novos alunos ao ano), sendo referência no cenário nacional. Este artigo relata o histórico da criação e expansão do Laboratório de Simulação (LabSim) e suas diversas experiências no decorrer dos anos.

\section{Metodologia}

Trata-se de revisão narrativa da evolução das experiências do LabSim utilizando busca e análise de registros e documentos, além de artigos e capítulos de livros referentes à temática. Para melhor detalhamento das ações este artigo foi dividido em temas, iniciando pela trajetória do LabSim e finalizando com perspectivas futuras.

\section{Trajetória do Laboratório de Simulação da Faculdade de Medicina da UFMG}

Em 1971, o Colegiado de Curso Médico da FM/UFMG começou a refletir sobre a reforma universitária e formas de elaborar uma nova estrutura de aprendizagem para o curso médico, pensando na necessidade de definir o perfil do profissional a ser formado, de forma a capacitá-lo para a inserção no mercado de trabalho, respeitando a nosologia prevalente em nosso meio.

Em 1994, houve a mudança do currículo médico e foram criadas as disciplinas Prática de Saúde A e B. Na época, a Escola de Enfermagem da UFMG assumiu em parte estas disciplinas, apoiando e interagindo com os alunos da graduação do curso médico. Em 1995, foi criado o Centro de Tecnologia Educacional em Saúde (CETES) na Faculdade de Medicina, já pensando em modernizar o ensino e trazer inovações tecnológicas para a FM/UFMG. O Prof. Cláudio de Souza assumiu a coordenação. Uma das primeiras iniciativas foi de reunir todos os modelos anatômicos e manequins dispersos em vários departamentos da faculdade e subutilizados, de forma a criar a cultura do ensino mediado pela simulação. A área física foi cedida pela diretoria da faculdade e o grupo de profissionais a configurar o recurso humano necessário para as práticas foi paulatinamente obtido, sob a supervisão do funcionário técnico administrativo Gilmar Tadeu de Azevedo Fidelis.

Gradativamente, foram adquiridos manequins e equipamentos de simulação para o LabSim. Em 2003, a professora Maria do Carmo Barros de Melo passou a atuar no CETES e em 2007, a professora foi designada para coordenar o LabSim. Foi elaborado um projeto para o Fundo Nacional de Saúde do Governo do Brasil e com isto o arsenal de manequins, equipamentos de simulação foram ampliados. A área física inicialmente era restrita, mas com a aquisição novos manequins e equipamentos de simulação, houve a reforma de uma ala da Faculdade de Medicina. Atualmente, o LabSim possui aproximadamente 600 metros quadrados de área física, com quatro salas equipadas com vidro unidirecional, som e vídeo de forma a permitir atividades de simulação avançada (com o professor gravando a atividade e fora do cenário de atendimento simulado). Possui também duas salas conjugadas para as práticas de habilidades de comunicação, também equipadas para gravação de som e voz. O almoxarifado permite que os manequins, equipamentos e material de consumo sejam guardados, de forma que todas as salas podem ser montadas para o cenário selecionado pelos docentes. O LabSim vem adquirindo paulatinamente manequins e equipamentos de simulação, alguns semi-robotizados e de simulação háptica (CETES, 2017).

A maior parte do acervo do LabSim foi adquirida por meio de financiamento do Fundo Nacional de Saúde, tendo como contrapartida a função de capacitar profissionais de saúde da rede pública para o desenvolvimento de competências e aquisição de habilidades, visando qualificar a assistência. Neste sentido, desenvolveu cursos de: capacitação de suporte básico de vida e em urgência e emergência; assistência e transporte do neonato; assistência à gestante e o neonato; prevenção e diagnóstico de câncer de mama e de colo de útero. Novas iniciativas estão em fase de planejamento. 
Atualmente, o LabSim participa de projetos públicos e privados, envolvendo diversas ações em simulação na área de saúde. Os recursos para a sustentabilidade do LabSim advêm da Diretoria da Faculdade de Medicina para os cursos de graduação e de projetos e consultorias na área de sua abrangência para os cursos de extensão ofertados.

A equipe do LabSim tem como missão "Criar, inovar e desenvolver ações envolvendo a simulação em saúde para aquisição de habilidades e desenvolvimento de competências, de forma ética, buscando adequada capacitação para qualificação da assistência."

\section{Atividades}

O As atividades que o LabSim desenvolve são amplas, envolvendo ambiente intra e extramuros na universidade. Vários projetos foram registrados como projetos de extensão. Os que mais destacaram foram: (1) "Salve a Vida com as mãos" - treinamento preconizado pela American Heart Association, que visa capacitar o leigo a prestar o primeiro atendimento ao paciente em parada cardiorrespiratória -, e está ativo desde 2016; (2) Curso de Urgência e Emergência pré-hospitalar - curso semipresencial (curso à distância utilizando vídeo em $2 \mathrm{D}$ e efeitos de animação) ofertado para 1000 médicos e enfermeiros da Secretaria Municipal de Saúde da Prefeitura Municipal de Belo Horizonte; (3) Curso de capacitaçãodos novos protocolos de síndrome coronariana aguda do Ministério da Saúde do Brasil, ocorreu em 2013; (4) Curso de capacitação em assistência neonatal para profissionais da rede estadual de saúde, realizado em 2012; (5) Curso Semipresencial de Primeiro Atendimento ao Trauma, para profissionais de saúde da UNIMED (plano de saúde privado brasileiro); (6) Curso de Suporte Básico de Vida para professores da Faculdade de Odontologia da Escola de Odontologia da UFMG, ocorrido em 2012.

Na graduação são várias são as disciplinas que utilizam o LabSim, em especial após a reforma curricular ocorrida a partir de 2014 (Tabela 1).

Tabela 1. Disciplinas, alunos, monitores atuantes no Laboratório de Simulação UFMG.

\begin{tabular}{|c|c|c|c|}
\hline Disciplinas & $\begin{array}{c}\mathbf{N}^{\circ} \text { alunos } \\
\text { envolvidos na } \\
\text { disciplina/semestre }\end{array}$ & $\begin{array}{c}N^{\circ} \text { monitores } \\
\text { do período de } \\
2014-2017 \\
\end{array}$ & $\begin{array}{c}N^{\circ} \text { professores } \\
\text { envolvidos/semestre }\end{array}$ \\
\hline $\begin{array}{l}\text { Estágio em Medicina de } \\
\text { Urgência e } \\
\text { Traumatologia }\end{array}$ & 160 alunos/semestre & \multirow{15}{*}{$\begin{array}{l}65 \text { monitores } \\
\text { (dentre esses, } \\
13 \text { bolsistas e } \\
52 \text { voluntários) }\end{array}$} & \multirow{8}{*}{$\begin{array}{l}\text { Número de professores, } \\
\text { especialidades: } \\
6 \text {, aparelho locomotor } \\
\text { 3, cirurgia (02 cirurgia } \\
\text { e } 01 \text { anestesiologia) } \\
\text { 15, pediatria (13 } \\
\text { pediatria } \\
\text { e } 02 \text { neonatologia) } \\
\text { 10, Ginecologia e } \\
\text { obstetrícia } \\
\text { 8, clínica médica. }\end{array}$} \\
\hline Técnicas Cirúrgicas & 60 alunos/semestre & & \\
\hline Clínicas Cirúrgicas & 160 alunos/semestre & & \\
\hline $\begin{array}{l}\text { Estágio em Ginecologia e } \\
\text { Obstetrícia }\end{array}$ & 60 alunos/semestre & & \\
\hline $\begin{array}{l}\text { Semiologia I e II (Módulo } \\
\text { de Clínica } \\
\text { Médica, Pediatria e } \\
\text { Otorrinoloaringologia) }\end{array}$ & 120 alunos/semestre & & \\
\hline $\begin{array}{l}\text { Internato em Pediatria } \\
\text { (módulo enfermaria } \\
\text { e neonatologia) }\end{array}$ & 120 alunos/semestre & & \\
\hline $\begin{array}{l}\text { Atendimento Pré- } \\
\text { hospitalar e Primeiros } \\
\text { Socorros }\end{array}$ & 160 alunos/semestre & & \\
\hline Pediatria I & 320 alunos/semestre & & \\
\hline Pediatria II & 150 alunos/semestre & & \\
\hline Pediatria V & 150 alunos/semestre & & \\
\hline Clínica Médica II & 120 alunos/semestre & & \\
\hline Ginecologia Básica & 120 alunos/semestre & & \\
\hline Obstetrícia Básica & 160 alunos/semestre & & \\
\hline $\begin{array}{l}\text { Suporte de Vida em } \\
\text { Urgência e Emergência }\end{array}$ & 320 alunos/semestre & & \\
\hline Estágio em Urgência e & 160 alunos/semestre & & \\
\hline
\end{tabular}

Revista Internacional em Língua Portuguesa, 2018, №33, Ciências da Saúde e Tecnologia, pp. 119-130 


\begin{tabular}{|l|l|l|l|}
\hline Emergência & \multirow{3}{*}{} & \\
\cline { 1 - 2 } $\begin{array}{l}\text { Internato optativo de } \\
\text { anestesiologia }\end{array}$ & 30 alunos/semestre & \\
\cline { 1 - 3 } $\begin{array}{l}\text { Liga de Simulação em } \\
\text { Saúde-LASS }\end{array}$ & 100 alunos/semestre & \\
\hline
\end{tabular}

Nas disciplinas Pediatria I, Pediatria II, Ginecologia Básica, Obstetrícia Básica, Internato de Pediatria, Internato de Clínica, Internato de Cirurgia, Estágio em Urgência e Emergência a avaliação formativa dos alunos ocorre por meio da utilização da metodologia do OSCE (Objective Structured Clinical Examination).

A área física do LabSim é modificada pontualmente para a execução do OSCE, de forma a disponibilizar ambientes simulados de acordo com o caso clínico e o cenário selecionados. O objetivo é avaliar as competências que foram desenvolvidas pelo aluno em cada disciplina. Os casos são apresentados e os alunos tem cinco minutos para demonstrar o que ele faria como médico do paciente. São utilizados manequins, atores equipamentos, computadores e dispositivos auxiliares. O número de estações varia de acordo com o número de alunos.

Para o desenvolvimento das atividades propostas, o Laboratório de Simulação agrega em sua equipe, acadêmicos do curso de medicina, participantes do programa de monitoria de graduação da UFMG. O programa visa dar suporte às atividades acadêmicas curriculares vinculadas aos projetos pedagógicos do curso de medicina, em seus departamentos. São oferecidas bolsas acadêmicas e a concessão das mesmas tem como objetivo contribuir para a melhoria na qualidade das disciplinas e as diversas ações envolvidas, iniciando o estudante nas atividades da docência. Os acadêmicos passam por processos de seleção em cada departamento envolvido. Os primeiros aprovados recebem uma bolsa da instituição. As demais vagas serão preenchidas por outros aprovadas, mas na condição de voluntários. A monitoria é uma atividade acadêmica, reconhecida como geradora de créditos, que serão posteriormente integralizados ao currículo e que contam pontos para as provas de residência médica.

Os monitores são supervisionados diretamente pelo professor coordenador do LabSim e de seus orientadores de cada disciplina. Planos de trabalho são elaborados pela coordenação e professores, para serem desenvolvidos pelos alunos. As atribuições dos acadêmicos no LabSim envolvem participação ativa nas atividades didáticas e de simulação, apoio aos docentes, participação nos treinamentos de capacitação e atualização, participação nas reuniões científicas e administrativas, preparo de salas, manequins e cenários para a condução das simulações clínicas. Elaboração de casos customizados para os softwares dos manequins de alta fidelidade, elaboração de guias de utilização dos manequins e equipamentos de simulação além da produção de material didático. É estimulada a participação em eventos de extensão, jornadas acadêmicas e congressos.

Além das atividades desenvolvidas pelos monitores no LabSim, existe a participação de graduandos de medicina e enfermagem da Liga Acadêmica de Simulação em Saúde (LASS). A liga foi formada em 2012 e tem como principal objetivo o estudo e a aplicação prática da Simulação na área de saúde com ênfase na interdisciplinaridade. A liga é supervisionada por um professor ou tutor, e é também considerada uma atividade geradora de créditos para o estudante. A liga desenvolve nos alunos a oportunidade de se tornarem ativos e autônomos na busca de conhecimentos específicos, de desenvolverem iniciativas e compartilhar experiências. Os acadêmicos participam de encontros semanais, com agenda diversificadas e multidisciplinares, auxiliando nas atividades do laboratório de simulação, como suporte para os docentes e monitores de graduação. Várias atividades são hoje desenvolvidas: ações de extensão, que incluem atividades com a comunidade, utilizando a simulação como ferramenta de ensino, em áreas de conhecimento afins, principalmente na área de urgência e emergência. Entre as pessoas da comunidade treinadas citamos grupos pertencentes à: escolas de ensino médio, graduação, pós graduação, comunidade trabalhadores da limpeza pública (garis). Nestes cenários, a experiência possibilitou reflexões sobre ao uso da simulação, e o trabalho em equipe e principalmente a integração multidisciplinar.

Um dos programas recentemente desenvolvidos pelo CETES- LabSim, foi a criação do Grupo de Interesse Especial Simulação em Saúde - (SIG SimSaúde), da Rede Universitária de Telemedicina (RUTE) vinculada ao Ministério da Ciência e Tecnologia e Inovação. A Rede RUTE é coordenada pela Rede Nacional de Ensino e Pesquisa.

Um dos objetivos da Rede RUTE é estimular a integração e a colaboração entre profissionais de saúde por meio de Grupos de Interesse Especial (do inglês Special Interest Groups - SIGs). Estes grupos promovem sessões, por videoconferência ou webconferência, para debates, discussões de caso, aulas, pesquisas e avaliações à distância, em 
várias especialidades e subespecialidades médicas, para diversos profissionais da área da saúde, em diversos níveis de formação profissional (RUTE, 2017).

O que impulsionou o CETES a criar este grupo foi a percepção de que a Simulação é, hoje, uma área do conhecimento que está em franca expansão nos diversos níveis de atenção à saúde e é reconhecidamente uma ferramenta metodológica eficaz para o ensino, assistência, treinamento, e ao mesmo tempo abre portas para um campo amplo de pesquisas. Além disto, tem um caráter multidisciplinar, possibilitando a aplicação e a conjugação de saberes e experiências de várias áreas da saúde: Medicina, farmácia, enfermagem, terapia ocupacional, sistemas de gestão, segurança do paciente, entre outras) envolvendo inovação tecnológica, habilidades de comunicação, questões bioéticas, além de possibilitar a integração e interação de profissionais interessados no ensino mediado por simulação. O SIG - Simulação em Saúde (SimSaúde) congrega várias instituições brasileiras vinculadas à Rede RUTE, em todo o Brasil. A principal meta é envolver e incentivar a participação de instituições de todo o Brasil, compartilhando experiências, conhecimentos e a diversidade de suas realidades. O Grupo de Interesse Especial em Simulação em Saúde, possui uma agenda anual, e seus encontros são mensais, sempre com a temática envolvendo simulação em saúde e suas interfaces. Nossos convidados são integrantes de instituições universitárias de Saúde e de várias categorias profissionais. Buscamos também, experiências internacionais em Simulação em Saúde, com convidados estrangeiros, em especial de Portugal.

O LabSim tem expandido suas ações para os ambientes reais de atendimento, como por exemplo, hospitais e pronto atendimento utilizados como cenários de prática dos acadêmicos do curso de medicina por meio da realização da simulação in situ. Esta metodologia de ensino tem sido implementada nos últimos anos pelas principais instituições, como forma de capacitar profissionais e graduandos da área de saúde. O relatório "Errar é humano: Construindo um Sistema de Saúde Seguro" do Institute of Medicine Committee on Quality of Health Care (1999) provocou discussão sobre os formatos de treinamento e de organização dos serviços de saúde com o objetivo de reduzir as lesões iatrogênicas e os eventos adversos, sendo a simulação indicada como uma forma de capacitar e promover educação continuada para os profissionais de saúde.

A simulação in situ é aquela que ocorre em ambiente real de trabalho, permitindo treinamento mais efetivo quando comparada com as simulações realizadas em ambiente de laboratório (Alkhulaif et al., 2016). Permite a reprodução da situação vivenciada em cenário real em que o profissional atua, interação entre a equipe assistencial, identifica os obstáculos à implementação dessas habilidades no ambiente em que o paciente está presente, proporcionando possibilidade de melhoria da infraestrutura do atendimento e melhor gestão do serviço de saúde (Saclabrini, Fonseca, \& Brandão, 2017).

A simulação in situ tem sido mais utilizada na disciplina "Estágio em Urgências", módulo pediatria, pelos alunos do décimo segundo período do curso de medicina. A sala de emergência dos campos de estágio é utilizada para o desenvolvimento das atividades. Os casos são previamente preparados de acordo com a nosologia prevalente e os protocolos do serviço. Check list são preparados baseados nos objetivos de ensino e o debriefing planejados. A experiência vem sendo realizada há um ano e meio, ocorrendo integração entre os alunos e os profissionais de saúde, de forma multidisciplinar. As atividades demandam 30 minutos de duração, sendo 10 minutos para o pré-cenário e apresentação do caso, 10 minutos para o atendimento simulado e 10 minutos para o debriefing. Desta forma, a demanda assistencial não é prejudicada. Um carrinho simulado similar ao real é disponibilizado e quando a sala de emergência está ocupada, uma sala próxima é utilizada. Algumas atividades são filmadas para discussão posterior e para tal um termo de consentimento esclarecido é assinado pelos participantes.

Para a criação de cenários, seja para as atividades desenvolvidas no LabSim ou in situ, inicialmente é necessário uma reflexão sobre qual é o objetivo de aprendizado da atividade a ser proposta. O objetivo deve ser claro e pontual de forma a evitar divagações. É preferível fazer mais casos do que elaborar casos complicados e com muitas informações, pois poderia confundir o participante. A partir daí, é necessário identificar qual a infraestrutura disponível (área física, manequins de baixa ou alta fidelidade, equipamentos, material de consumo, profissionais para atuarem como instrutores/facilitadores, atores). Um check list de tudo que for necessário para a montagem do cenário deve ser elaborado. A seguir, o caso-cenário deve ser discutido e um piloto com profissionais experientes deve ser conduzido. A atividade deve envolver pequenos grupos, sendo o número ideal 5 a 6 alunos ou profissionais (idealmente deve ser formada uma equipe multiprofissional), os quais devem utilizar crachá com nome visível. Antes do início da atividade, o pré-cenário, ou seja uma descrição de tudo que estará disponível para o desenvolvimento da atividade deve ser descrito. $O$ instrutor/facilitador deve fornecer os dados iniciais do caso/paciente para os participantes e os mesmos vão agir de acordo com funções pré-determinadas, como por exemplo líder, médico auxiliar, enfermeiro, entre outros. Exames complementares podem ser disponibilizados. Os pacientes podem evoluir bem ou não, de acordo com ações 
aceitáveis ou inaceitáveis que os participantes optarem como conduta. Se o manequim for de alta fidelidade, o sistema computadorizado pode ser adequado para respostas vitais favoráveis ou desfavoráveis, de acordo com a decisão tomada. O instrutor/ facilitador deve, preferencialmente, ficar fora do cenário e comandar por voz, se necessário, os participantes. Após o término do caso, o instrutor/facilitador e os participantes, devem realizar o debriefing do caso, e de preferência utilizar filmagens da atividade para substanciar a discussão.

A técnica de debriefing (Saclabrini, Fonseca, \& Brandão, 2017), palavra que em português poderia ser definida como análise reflexiva de uma atividade desenvolvida, tem sido muito utilizada nas práticas realizadas no LabSim e nas atividades de Simulação in situ. Propicia maior vivência emocional do caso/cenário e é muito útil para identificar os problemas relacionados ao trabalho em equipe, levando ao desenvolvimento de competências. Pode ser utilizada também após assistir um vídeo, o que denominamos videodebriefing, o que facilita o processo ensino aprendizado por ser utilizado para diversos grupos de alunos ou profissionais, evitando a montagem de cenários para cada prática.

Consiste de três fases: descritiva, analítica e aplicativa. Na fase descritiva, o ideal é que cada participante relate, sem crítica e sem juízo de valores, o que fez e o que o outro colega fez diante do caso/cenário apresentado. Na analítica, cada um descreve o que fez de bom e o que poderia fazer melhor da próxima vez. Na fase aplicativa, é sumarizado o que foi discutido diante do caso e das reflexões e o sintetizado o que poderia ser feito diante de um caso clínico semelhante em futuros atendimentos. Em muitos casos/cenários é possível identificar a vivência emocional de cada participante. Valores intrínsecos e extrínsecos podem interferir no processo ensino-aprendizagem. 0 instrutor/facilitador deve estar atento, saber escutar e dar suporte, quando necessário.

Para o ensino médico é muito importante o treinamento dos acadêmicos em habilidades de comunicação e o laboratório de simulação é o local apropriado para as práticas. Durante anos acreditou-se que a comunicação médicopaciente era adequada. Entretanto, evidências têm demonstrado o contrário. Inúmeras reclamações decorrentes de falhas na comunicação médico-paciente sugerem a necessidade de buscar ferramentas que viabilizem melhorias nesta situação (Stewart, 1995; Boon \& Stewart, 1998).

Depois de décadas de pesquisa em comunicação, que começaram em 1968 com Korsch e colaboradores, o treinamento de habilidades de comunicação ganhou atenção na educação médica. Em 1990, a habilidade de comunicação foi definida como uma das competências essenciais por parte do American Board of Accreditation of Medical Education e pelo Royal College of Physicians and Surgeons of Canada (Dielissen et al., 2012). Uma comunicação eficaz entre médicos, pacientes e famílias é essencial para a prestação de cuidados de saúde de qualidade. Reconhecendo isso, cada vez mais, o mundo ocidental tem tentado desenvolver e melhorar os currículos com vistas à adoção de formas mais eficazes de ensinar e avaliar habilidades de comunicação médica.

A simulação é uma técnica que evoca aspectos do mundo real em um ambiente interativo. Mais especificamente, a experiência clínica simulada objetiva replicar os aspectos essenciais de uma situação clínica, permitindo uma resposta adequada quando algo semelhante acontecer em um contexto real. Assim, a simulação clínica é uma ferramenta poderosa de aprendizado que pode ser aplicado em todos os níveis da educação médica, enfatizando a multidisciplinaridade em diversas situações clínicas (Gaba 2007; Brandão, Collares, \& Marin, 2014).

O emprego das técnicas de simulação permite que se ofereçam as mesmas oportunidades de aprendizado, prática e treinamento para todos os estudantes de forma mais homogênea, sem depender de circunstâncias e do acaso, envolvidos no aprendizado baseado em situações reais (Troncon, 2007). A simulação é feita em ambiente controlado, protegido, que permite ao participante errar e aprender com os seus erros. Além disso, existe a possibilidade de gravação das atividades simuladas realizadas e a utilização de vídeo-debriefing de cenários pré-elaborados, que contribuem para o auto desenvolvimento e podem ser objetos para análise do desempenho do aluno,.

Manequins ou atores podem utilizados como pacientes padrões. O uso de pacientes simulados é uma estratégia baseada em uma forma ativa de aprendizagem, que se mostra mais eficaz do que estratégias puramente teóricas (Lane \& Rollnick, 2007; Haeseler et al., 2011; Bouter, van Weel-Baumgarten, \& Bolhuis, 2013; Bell et al., 2014).

No contexto da renovação dos métodos de pesquisa utilizados na pós-graduação na UFMG foi montado um Laboratório de Treinamento em Habilidades de Comunicação, integrado ao LabSim. Este laboratório é pioneiro na Faculdade de Medicina da UFMG, e está incorporado ao patrimônio da Universidade representando um legado do projeto CAPES PRÓ-ENSINO NA SAÚDE (Edital 024/2010 - Projeto 1606/2011) para desenvolvimento de atividades curriculares formais, de ensino e pesquisa, aos alunos dos cursos de graduação e pós-graduação da Faculdade de Medicina. 
O campo de pesquisa em habilidades de comunicação é muito vasto, com aplicação em diversos cenários de aprendizagem, podendo ser estendido a outras profissões, pois trata-se de habilidade essencial para quem trabalha com pessoas.

\section{Perspectivas Futuras}

O LabSim atualmente dispõe de área física pequena em relação ao número de usuários, mas, existe um projeto aprovado, aguardando liberação de recurso financeiro para construção de nova área. A nova área é composta de várias salas para debriefing, simulação avançada, auditório e é acoplada em salas específicas para o desenvolvimento das atividades relacionadas ao OSCE. A simulação vem sendo gradativamente incorporada às atividades desenvolvidas na faculdade e, em breve, deverá ser ampliado o número de docentes e discentes envolvidos. Tem havido aproximação com a Residência Médica do Hospital Universitário, com participação de residentes e estudantes da graduação em atividades conjuntas. A comunidade em geral vem sendo envolvida com ações de treinamento e capacitação, em especial ao que se refere à reanimação cardiorrespiratória e primeiro atendimento aos pacientes graves. Pesquisas vem sendo desenvolvidas. O LabSim da Faculdade de Medicina é atualmente referência em capacitações de profissionais de instituições de saúde e de escolas privadas de cursos da área de saúde. O SIG SimSaúde tem destaque no cenário nacional, permitindo a troca de experiência entre os profissionais interessados e em breve pretendemos ampliar o número de atividades e de participações, inclusive de instituições de outros países. Com as novas tecnologias de informação e comunicação, a simulação deverá crescer e ampliar o seu campo de atuação.

\section{Referências}

1999. To Err is Human: Building a Safer Health System. In: National Academy of Sciences Engineering Medicine. http://nationalacademies.org/hmd/ /media/Files/Report\%20Files/1999/To-Err-is-Human/To\%20Err\%20is\%20Hum an\%201999\%20\%20report\%20brief.pdf.

Alkhulaif, A., Julie, I., Barton, J., Nagle, E., Yao, A., Clarke, S., Venugopal, S., Hammontree, W., Ramirez, J., Dunbar, K., Sebat, C., Melo, M. C., \& Bair, A. (2016). In-Situ Simulation Training: Advantages, Challenges and Obstacles." Latin American Journal of Telehealth 3(2), 6-13.

Bell, S. K., Pascucci, R., Fancy, K., Coleman, K., Zurakowski, D., \& Meyer, E. C. (2014). The educational value of improvisational actors to teach communication and relational skills: Perspectives of interprofessional learners, faculty, and actors. Patient education and counseling 96(3), 381-388.

Boon, H., \& Stewart, M. (1998). Patient-physician communication assessment instruments: 1986 to 1996 in review. Patient education and counseling 35(3), 161-176.

Bouter, Shifra, Evelyn van Weel-Baumgarten, and Sanneke Bolhuis. (2013). Construction and validation of the nijmegen evaluation of the simulated patient (nesp): assessing simulated patients' ability to role-play and provide feedback to students. Academic Medicine 88(2), 253-259.

Brandão, C. F., Collares, C. F., \& Marin, H. F. (2014). A simulação realística como ferramenta educacional para estudantes de medicina. Sci Med 24(2), 187-92.

Brasil. 2014. Resolução N. 3, de 20 de junho de 2014. Diretrizes Curriculares Nacionais do Curso de Graduação em Medicina. Accessed 01/05/2017. Retrieved from http://portal.mec.gov.br/index.php?option=com_docman\&amp;am p;view=download\&amp;amp;alias=15874-rces003-4\&amp;amp;category_slug=junho-2014-pdf\&amp;amp;Itemid=30 192.

CETES. (2017). Centro de Tecnologia em Saúde. Faculdade de Medicina da Universidade Federal de Minas Gerais. UFMG, accessed 30/05/2017. http://site.medicina.ufmg.br/cetes/laboratorio-simulacao/.

Dent, J., Harden, R., \& Hunt, D. (2017). A practical guide for medical teachers. 5 ed. ed. London: Elsevier Health Sciences.

Dielissen, P., Verdonk. P., Bottema, B., Kramer, A., \& Lagro-Janssen, T. (2012). Expert consensus on gender criteria for assessment in medical communication education. Patient education and counseling 88(2), 189-195. 
Gaba, David M. 2007. “The future vision of simulation in healthcare." Simulation in Healthcare2 (2):126-135.

Haeseler, F., Fortin, A. H., Pfeiffer, C., Walters, C., \& Martino, S. (2011). Assessment of a motivational interviewing curriculum for year 3 medical students using a standardizedpatient case. Patient education and counseling 84(1), 2730.

Korsch, B. M., Gozzi, E. K., \& Francis, V. (1968). Gaps in doctor-patient communication. Pediatrics 42(5), 855-871.

Lane, C., \& Stephen R. (2007). The use of simulated patients and role-play in communication skills training: a review of the literature to August (2005). Patient education and counseling 67(1), 13-20.

Martins, J. C., Mazzo, A., Baptista, R. C., Coutinho, V. R., Godoy, S. G., Mendes, I. A., \& Trevizan, M. A. (2012). A experiência clínica simulada no ensino de enfermagem: retrospectiva histórica. Acta paul enferm 25(4), 619-25.

RUTE. (2017). Rede Universitária de Telemedicina do Brasil (Sig RUTE). Accessed 20/05/2017. Retrieved from http://rute.rnp.br/web/rute/sigs.

Saclabrini, A., Fonseca, A., \& Brandão, C. (2017). Simulação realística e habilidades na saúde. Rio de Janeiro: Atheneu.

Stewart, M. A. (1995). Effective physician-patient communication and health outcomes: a review. CMAJ: Canadian Medical Association Journal 152(9), 1423.

Troncon, L. A. (2007). Utilização de pacientes simulados no ensino e na avaliação de habilidades clínicas. Medicina (Ribeirao Preto. Online) 40(2), 180-191.

\section{Sobre os Autores}

Maria do Carmo Barros de Melo (PhD) possui pós-doutoramento. É professora Associada do Departamento de Pediatria e coordenadora do Laboratório de Simulação da Faculdade de Medicina da Universidade Federal de Minas Gerais, Brasil.

Monalisa Maria Gresta (MsC) é professora convidada do Laboratório de Simulação da Faculdade de Medicina da Universidade Federal de Minas Gerais, Brasil. Enfermeira. Mestre em Enfermagem.

Nara Lucia Carvalho e Silva é professora convidada do Laboratório de Simulação da Faculdade de Medicina da Universidade Federal de Minas Gerais, Brasil. Enfermeira, mestranda em Ciências da Saúde/área de concentração da Saúde da Criança e do Adolescente.

Gimar Fidelis é psicólogo e professor convidado da Faculdade de Medicina da Universidade Federal de Minas Gerais, Brasil.

Maria Angélica Tibaes é enfermeira e funcionária do Laboratório de Simulação da Faculdade de Medicina da Universidade Federal de Minas Gerais, Brasil.

Josemar de Almeida Moura é Doutor e professor Adjunto da Faculdade de Medicina da Universidade Federal de Minas Gerais, Brasil.

Claudio de Souza é Doutor, professor Associado e coordenador do centro de Tecnologia em Saúde da Faculdade de Medicina da Universidade Federal de Minas Gerais, Brasil. 